\title{
Comments on : Accuracy of Raman lidar water vapor calibration and its applicability to long-term measurements
}

David N. Whiteman, NASA/GSFC, Greenbelt, MD 20771, david.n.whiteman@nasa.gov

Demetrius Venable, Howard University, Washington, DC

Eduardo Landulfo, IPEN, Sãu Paolo, Brazil 


\section{Abstract}

In a recent publication, LeBlanc and McDermid[Leblanc and McDermid, 2009] proposed a hybrid calibration technique for Raman water vapor lidar involving a tungsten lamp and radiosondes. Measurements made with the lidar telescope viewing the calibration lamp were used to stabilize the lidar calibration determined by comparison with radiosonde. The technique provided a significantly more stable calibration constant than radiosondes used alone. The technique involves the use of a calibration lamp in a fixed position in front of the lidar receiver aperture. We examine this configuration and find that such a configuration likely does not properly sample the full lidar system optical efficiency. While the technique is a useful addition to the use of radiosondes alone for lidar calibration, it is important to understand the scenarios under which it will not provide an accurate quantification of system optical efficiency changes. We offer examples of these scenarios. 


\section{Introduction}

The Network for the Detection of Atmospheric Composition Change (NDACC) has recently established long term monitoring of water vapor using Raman lidar as one of its core objectives [Leblanc and McDermid, 2009]. Other international efforts such as the Global Climate Observing System (GCOS) Reference Upper Air Network (GRUAN) [Seidel et. al., 2009] are tasked with the same objective. One of the paramount needs for developing a long term dataset for monitoring atmospheric trends is a calibration that varies randomly around some mean value and does not involve step jumps of unknown magnitude [Weatherhead et. al., 1997]. These step jumps in calibration increase the time required to detect atmospheric trends which is already typically measured in decades [Weatherhead et. al., 1998] [Boers and Meijgaard, 2009]. For this reason it is important to carefully examine any calibration techniques developed for ensuring stable, long-term calibrations. Here we examine the hybrid lamp technique proposed by Leblanc and McDermid [Leblanc and McDermid, 2009] (hereafter referred to as LM2009) for calibration of Raman water vapor lidar. 


\section{The calculation of water vapor mixing ratio from Raman}

\section{lidar}

The water vapor mixing ratio can be expressed as [Whiteman 2003a] [Whiteman 2003b]:

$$
w=k \frac{O_{N}(r)}{O_{H}(r)} \frac{F_{N}(T)}{F_{H}(T)} \frac{P\left(\lambda_{H}, r\right)}{P\left(\lambda_{N}, r\right)} \frac{\frac{d \sigma_{N}(\pi)}{d \Omega}}{\frac{d \sigma_{H}(\pi)}{d \Omega}} \frac{\xi\left(\lambda_{N}\right)}{\xi\left(\lambda_{H}\right)} \Delta \tau\left(\lambda_{N}, \lambda_{H}, r\right)
$$

where $k$ is a factor determined by molecular weights and volume mixing ratios and is $\approx 0.485 . O_{X}(r)$ represents the channel overlap function as a function of range, $r$ for channel $X$, which, in this case, would be either the nitrogen, $N$, or water vapor channel, $H . F_{X}(T)$ is a temperature dependent factor that accounts for the temperature dependence of Raman scattering. $P\left(\lambda_{X}, r\right)$ is the backscattered power (after subtracting any background contribution due, for example, to skylight or detector noise). $\xi\left(\lambda_{X}\right)$ represents the total lidar receiver optical efficiency, $\xi$, at the laser wavelength, $\lambda_{X}$, and includes factors such as the reflectivity of the telescope, the transmission of any conditioning or wavelength selection optics, the transmission of any filters and the quantum efficiency of the detector. $\frac{d \sigma_{X}(\pi)}{d \Omega}$ represents the differential Raman backscattering cross section, $\Delta \tau\left(\lambda_{N}, \lambda_{H}, r\right)$ represents the atmospheric differential transmission at the water vapor and nitrogen wavelengths. 
The term of interest in equation 3.1 is the efficiency ratio, $\frac{\xi\left(\lambda_{N}\right)}{\xi\left(\lambda_{H}\right)}$, which includes the transmission or reflection efficiency of all optical components and, given that reflection and transmission efficiencies can change as a function of position on each optical component, is a weighted average of the portions of these components in use. We will first consider the use of a calibration lamp in a fixed position in front of the telescope and then discuss the influence of position-dependent efficiency changes.

\section{The use of a calibration lamp in a fixed position}

The technique proposed in LM2009 involves a tungsten calibration lamp powered by a stabilized current source. The use of a calibration lamp is very attractive as a component of a Raman water vapor lidar calibration technique since the quantity that must be evaluated with accuracy is the ratio of the transmission efficiencies at the Raman water vapor $(407.5 \mathrm{~nm})$ and nitrogen $(386.7 \mathrm{~nm})$ wavelengths. An examination of $200 \mathrm{~W}$ calibration lamps obtained over a period of several years indicated that the effective color temperature of these lamps varied typically within a range of $\pm 10-20 K$ [Optronics Laboratories, 2009]. This color temperature range implies different spectral outputs for the lamps which, for the small spectral range of 386.7 - $407.5 \mathrm{~nm}$, can be approximated with high accuracy 
by the Planck black body formula. Evaluating the Planck formula at 386.7 and $407.5 \mathrm{~nm}$ for changes in lamp color temperatures of $\pm 20 \mathrm{~K}$ indicates that the ratio of spectral outputs at these two wavelengths varies by $0.3 \%$ or less. This implies that the use of uncalibrated tungsten lamps likely supplies a spectral reference for the ratio of water vapor and nitrogen channel optical efficiencies that is accurate to better than $0.3 \%$. The use of a tungsten lamp with calibration traceable to NIST standards to within $2 \%$ reduces the uncertainty in this ratio to $\sim 0.1 \%$. Therefore, the calibration lamps provide a highly stable reference for use in a Raman water vapor lidar calibration. But the primary question to be addressed here is whether the full lidar system efficiency is well represented by using a lamp at a fixed position in front of the lidar receiver.

Figure 11.1 shows the ray trace of a calibration lamp placed in front of a telescope and receiver system. The lamp is positioned near the edge of the primary aperture and emits in all directions. By virtue of a field stop used at the prime focus of the telescope, only those rays that diverge from the optical axis within an angle of \pm one half the angular field of view will be accepted by the field stop. The right of figure 11.1 is shows the pattern of rays that will be transmitted fully through the optical train of the lidar system due to this angular filtering.

This illustrates the fundamental concern of using a lamp at a fixed position 
in front of the telescope aperture. Only a very small fraction of the telescope primary mirror and all optics that follow it in the optical train are sampled by the direct beam from the lamp. LM2009 discuss performing these measurements with the hatch that protects the lidar system closed. The light from the lamp is able to reflect off of the hatch cover and thus illuminate the entire telescope. The use of this technique raises some questions, however. What is the spectral response of the hatch cover and how stable is this response (i.e. is the hatch kept clean)? One can observe from figure 8 of LM2009 that closing the hatch changes the measured ratio by about $15 \%$. This implies that the reflectivities of the hatch at $407.5 \mathrm{~nm}$ and $386.7 \mathrm{~nm}$ are not the same. So the spectral characteristics of the light that is reflected off of the hatch is significantly different than the calibrated light that enters the telescope directly from the lamp.

Another question is: what fraction of the entire signal sampled by the telescope is due to the direct beam versus the signal that is reflected off of other surfaces? In an attempt to address this question, an experimental configuration similar to that illustrated in figure 5 from LM2009 was established using the Howard University Raman Lidar in Beltsville, MD. The calibration lamp was placed approximately 1 meter away from the telescope and a white reflective board was placed approximately $30 \mathrm{~cm}$ above the lamp. An examination of the signal intensity with and 
without this reflective board in place indicated that approximately $90 \%$ of the signal was due to the direct beam and only $10 \%$ from the refelected beam. These results indicate that even the use of a reflective hatch can yield a telescope illumination that is strongly biased by the efficiency characteristics of the small fraction of the optical train illuminated by the forward beam as illustrated in Fig 1. The use of a reflective hatch may not evenly fill the telescope aperture and the component of the lamp output that is reflected from other surfaces will likely not retain the spectral characteristics of the lamp.

The uneven illumination of the telescope shown in figure 11.1, as apparent from the results of the experiment just described, propagates through the entire optical train to the detectors. If there are position dependent optical efficiencies in any of the optical components, the small fraction of the telescope sampled by the lamp may not properly represent the efficiency of the entire optical train. This statement pertains to a bulk optics configuration where the signal focussed by the telescope is collimated using traditional optics. Does the use of an optical fiber at the prime focus of the telescope improve this situation? Even with an optical fiber at the prime focus of the telescope, the experiment just described indicates that the efficiency of the telescope primary and secondary (if used) will be heavily weighted by the small spot illuminated by the lamp's forward beam. This effect is 
not changed by the use of a fiber. But does a fiber help to scramble the signal for the rest of the optical train removing concern about position dependent efficiency changes elsewhere in the optical train?

To investigate this, an experiment was performed again on the Howard University Raman lidar with the reflective board mentioned above in place. A calibration lamp was moved along a line from one edge of the telescope receiver to the other and the pattern emitted by an optical fiber placed at the prime focus of the telescope was recorded by training the light from the fiber on a screen and photographing the screen. Figure 11.2 shows the results of this experiment.

There are several things to note in figure 11.2. First the output of the fiber shows one or a set of modes being excited in the multi-mode fiber by the calibration lamp. As the position of the lamp changes, the mode pattern changes as well. The optical information from a small portion of the telescope is converted into a ring of varying diameter depending on the fiber modes in use. This is just a single experiment on a particular multi-mode fiber but it indicates that fiber optics do not in general scramble the optical signal sufficiently to remove concern about the position dependence of efficiences in the optical train. 


\section{Position dependence of optical efficiencies in the lidar optical system}

The preceding discussion strives to make the point that a calibration technique based on the use of a calibration lamp in a fixed position in front of a telescope will not equally sample the telescope or receiver optics. In the experiment described above, even with a reflective surface above the telescope the signal arriving at the prime focus of the telescope was dominated by the forward beam from the lamp. If the optical efficiency of some section of the primary or secondary telescope, either not sampled by the lamp or under-sampled by the lamp, changes over time then the results of the lamp calibration will not accurately reflect these changes. Also, if some debris were to fall on the telescope just at the point where the forward beam is making its intense spot a change in the efficiency ratio would likely be quantified where such a change did not represent the majority of the telescope aperture.

The possibility of position dependent changes in the optical efficiency of components of the optical train are not limited to the primary or secondary mirrors. For example, photomultiplier tubes [Simeonov et. al., 1999] and interference filters, commonly used in lidar receiver optical configurations, are known to be 
capable of position-dependent efficiency changes. An example of the position dependence of the transmission efficiency and bandpass of a narrow band interference filter is given in figure 11.3. The transmission of the 2 " diameter filter was determined with a beam approximately 0.5 inch in diameter. The transmission was quantified with the beam centered on the filter and offset approximately 0.5 inch from the center. The tranmission is observed to increase significantly at this offset position. A technique that fully samples the telescope aperture can be used to circumvent the position-dependent concerns described here. The next section illustrates such a technique.

\section{Scanning the full aperture of the lidar receiver}

A tungsten calibration lamp offers a highly stable ratio of output intensities at the water vapor and nitrogen wavelengths. The problems illustrated above come about by undersampling the telescope aperture using such a lamp. A technique involving movable $\mathrm{X}-\mathrm{Y}$ stages that can permit the lamp to scan the full aperture of a receiver telescope has been under development since 2006 as a joint project involving Howard University, IPEN in Brazil and NASA/GSFC [Torres et. al., 2008] [Landulfo et. al., 2009]. The calibration lamp is moved in small, even increments across the full useful aperture of the receiving telescope. At each location, the 
signals in the water vapor and nitrogen channels are quantified by the lidar data acquisition system using equal sampling time at each location. The ratio of the water vapor and nitrogen signals are then determined as a function of position. The efficiency ratio that characterizes the full optical system is then taken to be the average of all of the individual ratios after excluding data subject to edge effects (where not all of the lamp is directly illuminating the telescope primary). A photograph of the ganged X-Y stages in position above the HURL transmission window and an example of the scanned data obtained is shown in figure 11.4. A histogram of the 193 individual cells that constitute a full aperture scan is shown in figure 11.5. The mean value of the individual cells is $1.157 \pm 0.013$. The range of efficiency ratios is 1.116 to 1.190 indicating approximately a $7 \%$ variation in the measured efficiency ratio over the useful aperture of the telescope.

\section{Failure modes of a calibration lamp}

Even though scanning the full lidar telescope aperture with a calibration lamp provides an improved characterization of the full lidar receiver optical efficiency when compared with using a lamp in a fixed position, there are still "failure modes" that both techniques share in common that will now be described. Consider that the Raman return signals for water vapor and nitrogen excited in the atmosphere 
by a narrow-band laser have a small spectral width on the order of $0.1-0.2 \mathrm{~nm}$. Interference filters in use in Raman water vapor lidar measurements range from $0.1-1.0 \mathrm{~nm}$ typically and exhibit significant transmission variation over their nominal bandpass. These filters are angle sensitive so that small changes in the tilt angle of the filter can result in significant changes to the transmission efficiency of the desired Raman signal. However, the output of a calibration lamp is nearly uniform for the same small changes in wavelength. This implies that if a filter angular displacement were to occur, that the lamp technique would not properly account for the change in effective efficiency ratio. A similar statement could be made with respect to the slit position if a grating spectrometer is used to select the bandband.

To illustrate this, an experiment was performed using the NASA/GSFC ALVICE lidar system. Daytime measurements of water vapor mixing ratio were made on April 24, 2009 using 2 minute summations at various tilt angles ranging from 2 - 6 degrees for a $0.1 \mathrm{~nm}$ Raman $\mathrm{N}_{2}$ filter. (The peak transmission for the filter in use was obtained at a filter tilt angle of approximately 1-2 degrees). A tilt of 1 degree changes the center wavelength position of the filter by approximately $0.02 \mathrm{~nm}$, or about $20 \%$ of the bandwidth, so a significant change in transmission efficiency of the filter is expected. For this exercise, the mixing ratio values above 
the boundary layer were assumed constant over the approximate 1 hour that was required to perform the experiment. A different normalization value was used for each profile to achieve best agreement among all the profiles above the boundary layer. The normalization values used are plotted in figure 11.6 and range from 0.25 to 140 indicating that the nitrogen filter transmission changes by more than 2 orders of magnitude over this set of tilt angles. At the same time, the efficiency ratio recorded at each angle setting using the calibration lamp changes by less than $10 \%$. The lamp calibration is not useful to detect changes in the center wavelength of the filter which could be caused in the short term by a mechanical disturbance of the filter or in the long term by a filter degradation.

Another failure mode of either lamp calibration technique is also a failure mode of all the dominant calibration techniques, when measurements extending into the dry upper troposphere are considered. The calibration approaches of simple radiosonde matching in the lower to middle troposphere [Leblanc and McDermid, 2009], radiosonde + calibration lamp [Leblanc and McDermid, 2009], total column water scaling [Turner et. al., 2002], calibration assuming saturation at cloud base [Whiteman et. al., 2001], or absolute calibration efforts [Landulfo et. al., 2009] will not detect errors in Raman water vapor lidar mixing ratio measurements in the upper troposphere due to such effects as signal-induced-noise or fluo- 
rescence [Sherlock et. al., 1999] [Leblanc et.al., 2008]. To guard against errors created by effects such as these, comparison of the final lidar profile of water vapor with another instrument such as cryogenic frostpoint hygrometer or well-validated satellite measurements such as the Aura Microwave Limb Sounder [Vömel et. al., 2007b] will likely need to be done on a periodic basis.

\section{Discussion and Conclusion}

A tungsten calibration lamp provides a very stable ratio of outputs at the Raman water vapor and nitrogen wavelengths used for Raman water vapor lidar measurements of water vapor mixing ratio. Such a lamp has been found useful for quantifying the efficiency ratio at these two wavelengths as a method for improving on the technique of calibrating Raman water vapor lidar with respect to radiosondes [Leblanc and McDermid, 2009] (LM2009). This technique uses a calibration lamp at a fixed position in front of the telescope aperture. As discussed in LM2009, this technique can be useful in combination with radiosonde data to distinguish variations in calculated calibration coefficients that may be due to atmospheric variation instead of lidar system efficiency variation. Essentially, the technique is useful for detecting short term changes in the optical receiver system under the circumstances where the small fraction of the optical train that 
is sampled is representative of the entire system efficiency. As such, it may well form a component of the Raman water vapor lidar calibration technique that is currently under discussion within NDACC. We have focussed here on issues that can be presented by using a lamp in such a manner as a method of quantifying the ratio of optical efficiencies of the lidar channels. The uneven sampling of the lidar optical system aperture makes the technique susceptible to certain errors that do not plague traditional calibration approaches. If, for example, a small piece of debris fell onto the telescope at just the location where the calibration lamp makes its intense spot in the forward direction onto the primary mirror, a spurious calibration result could be obtained. Because of this, changes in calibration that are indicated by the fixed-lamp technique would need to be further investigated as to their source instead of assuming that they necessarily are due to an overall lidar system optical efficiency change. A technique involving 3 calibration lamps used in fixed positions in front of the telescope could provide sufficient redundancy to detect anomalous efficiency ratio measurements. The influence of changes in the center wavelength of the bandpass filters, such as could result if the receiver optics were mechanically disturbed, cannot be detected using lamp based techniques. To ensure that no such disturbance has occurred, it will be necessary to perdiocally check that the transmission features of the filters or spectrometer in use are prop- 
erly aligned with the Raman spectral features. Finally, there is a failure mode that is shared by lamp based calibration techniques and more traditional ones as well when considering measurements of upper tropospheric or lower stratospheric water vapor. Small amounts of signal-induced-noise or fluorescence can contaminate these high altitude measurements in a manner that could be detected only through a direct comparison with an external source believed to be accurate at these high altitudes.

\section{Acknowledgements}

The authors wish to acknowledge the support of the NASA Atmospheric Composition Program for support of these efforts.

\section{References}

\section{References}

[Avila et. al., 2004]

Avila, G., J. M. Fernández, G. Tejeda, S. Montero, The Raman spectra and cross-sections of $\mathrm{H} 2 \mathrm{O}$, $\mathrm{D} 2 \mathrm{O}$, and $\mathrm{HDO}$ in the $\mathrm{OH} / \mathrm{OD}$ stretching regions, J. Molec. Spectr., 228 38-65, 2004. 
[Boers and Meijgaard, 2009] Boers, R., E. Meijgaard, 2009: What are the demands on an observational program to detect trends in upper tropospheric water vapor anticipated in the 21st century, GRL, in press.

[Eyring et. al., 2007] V. Eyring et. al., 2007: Multimodel projections of stratospheric ozone in the 21st century, J. Geophys. Res., D16303, doi:10.1029/2006JD008332.

[Oman et. al., 2008] Oman, L., D. Waugh, S. Pawson, R. Stolarski, J. Nielsen, 2008: Understanding the Changes of Stratospheric Water Vapor in Coupled Chemistry-Climate Model Simulations, J. Atmos. Sci., 65, $3278-3291$.

[Landulfo et. al., 2009] E. Landulfo, R. F. Da Costa, A. S. Torres, F.J.S. Lopes, D. N.Whiteman, D. D. Venable, 2009: Raman water vapor lidar calibration, Proc. SPIE, Vol. 7479 .

[Leblanc et.al., 2008] Leblanc, T., I. S. McDermid, R. A. Aspey, FirstYear Operation of a New Water Vapor Raman Li- 
dar at the JPL Table Mountain Facility, California, 2008: J. Atmos. Ocean. Tech., Vol 25, 1454 $-1462$.

[Leblanc and McDermid, 2009] Leblanc, T., I. S. McDermid, 2009: Accuracy of Raman lidar water vapor calibration and its applicability to long-term measurements, Appl. Opt., 47, 30, $5592-5603$.

[Optronics Laboratories, 2009] Private Communication, Optronics Laboratories, 2009

[Seidel et. al., 2009]

Dian J. Seidel, Franz H. Berger, Howard J. Diamond, John Dykema, David Goodrich, Franz Immler, William Murray, Thomas Peterson, Douglas Siststerson, Michael Sommer, Peter Thorne, Holger Vömel, and Junhong Wang, Reference Upper-Air Observations For Climate: Rationale, Progress, and Plans, Bull. Amer. Meteor. Soc., March, 2009. 
[Sherlock et. al., 1999]

Vanessa Sherlock, Anne Garnier, Alain Hauchecorne, and Philippe Keckhut, 2009: Implementation and validation of a Raman lidar measurement of middle and upper tropospheric water vapor, Appl. Opt.,Vol. 38, No. 27, 5838 5858.

[Simeonov et. al., 1999]

Simeonov, V., G. Larcheveque, P. Quaglia, H. van den Bergh, B. Calpini, "Influence of the photomultiplier tube spatial uniformity on lidar signals, Appl. Opt., 38, 24, 5186 - 5190 (1999).

[Soden et. al., 2005]

Soden BJ, Jackson DL, Ramaswamy V, M. D. Schwarzkopf, X. Huang, 2005: The radiative signature of upper tropospheric moistening, Science, $310,5749,841-844$.

[Torres et. al., 2008]

Torres, A. S., Landulfo, E., Whiteman, D. N., and Venable, D., "Water vapor raman lidar independent calibration," in Reviewed and revised papers presented at the 24th International Laser Radar 
Conference, 23-28 June 2008, Boulder, Colorado, USA, Organizing Committee of the 24th International Laser Radar Conference (2008).

[Turner et. al., 2002]

D. D. Turner, R. A. Ferrare, L. A. H. Brasseur, W. F. Feltz, "Automated retrievals of water vapor and aerosol profiles from an operational Raman lidar", J. Atmos. Ocean. Tech., 19 (1) 37-50 (2002).

[Vömel et. al., 2007]

Vömel H., D. E. David, K. Smith, 2007: Accuracy of tropospheric and stratospheric water vapor measurements by the cryogenic frost point hygrometer: Instrumental details and observations, J. Geophys., Res, 112, D08305.

[Vömel et. al., 2007b]

Vömel, H., J. E. Barnes, R. N. Forno, M. Fujiwara, F. Hasebe, S. Iwasaki, 2007:Validation of Aura Microwave Limb Sounder water vapor by balloonborne Cryogenic Frost point Hygrometer measurements, J. Geophys. Res., D112, D24S37. 
[Weatherhead et. al., 1997] Weatherhead, E. C., G. C. Tiao, G. C. Reinsel, J. E. Frederick, J. J. DeLuisi, D. Choi, and W.-K. Tam, Analysis of long-term behavior of ultraviolet radiation measured by Robertson-Berger meters at 14 sites in the United States, J . Geophys Res., 102, 8737-8754, 1997.

[Weatherhead et. al., 1998] Weatherhead, E. C., G. C. Reinsel, G. C. Tiao, X-L Meng, D. Hoi,W-K Cheang, T. Keller, J DeLuisi, D. J. Wuebbles, J. B. Kerr, A. J. Miller, S. J. Oltmans, J. E. Frederick, Factors affecting the detection of trends: Statistical considerations and applications to environmental data, J. Geophys. Res., 103, D14, 17,149-17,161, 1998.

[Whiteman et. al., 2001] Whiteman, D.N., K. D. Evans, B. Demoz, D. O'C. Starr, E. Eloranta, D. Tobin, W. Feltz, G. J. Jedlovec, S. I. Gutman, G. K. Schwemmer, M. Cadirola, S. H. Melfi, F. J. Schmidlin, 2001: Raman lidar measurements of water vapor and cirrus 
clouds during the passage of hurricane Bonnie, $J$. of Geophys. Res., 106, No. D6, 5211-5225.

[Whiteman 2003a] Whiteman, David N, Examination of the traditional Raman lidar technique. I. Evaluating the temperature-dependent lidar equations, Applied Optics, 42, No. 15, 2571-2592 (2003).

[Whiteman 2003b] Whiteman, David N., Examination of the traditional Raman lidar technique. II. Evaluating the ratios for water vapor and aerosols, Applied Optics, 42, No. 15, 2593-2608 (2003). 

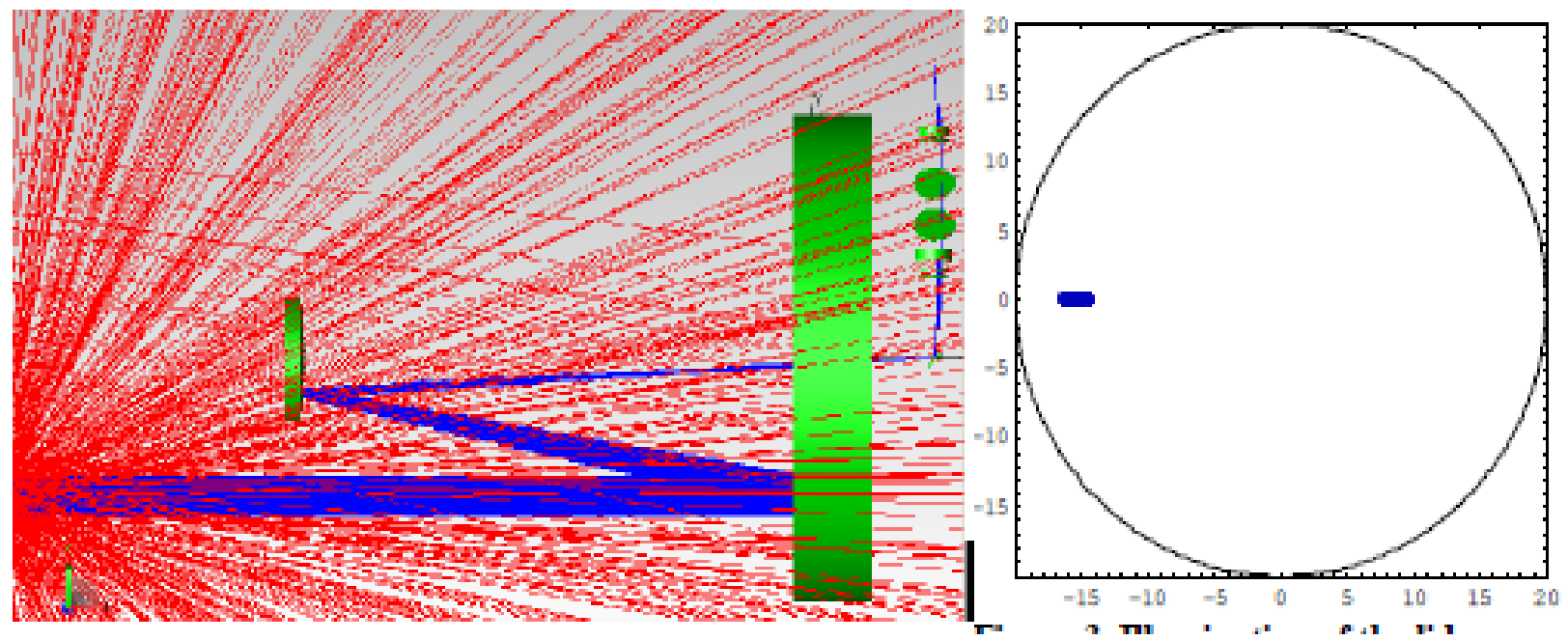

Figure 11.1: Ray trace of a spectral lamp at a fixed position in front of a telescope system. (Left) The red rays indicate the lamp output in all directions. The blue rays are those that fall within the angular field of view of the telescope system and are thus permitted to propagate down the lidar optical train. (Right) The blue rays that are accepted by the optical system are only those that fall within +/fov $/ 2$ of a line parallel to the optical axis that is directly in front of the calibration lamp position.

\section{Figures}




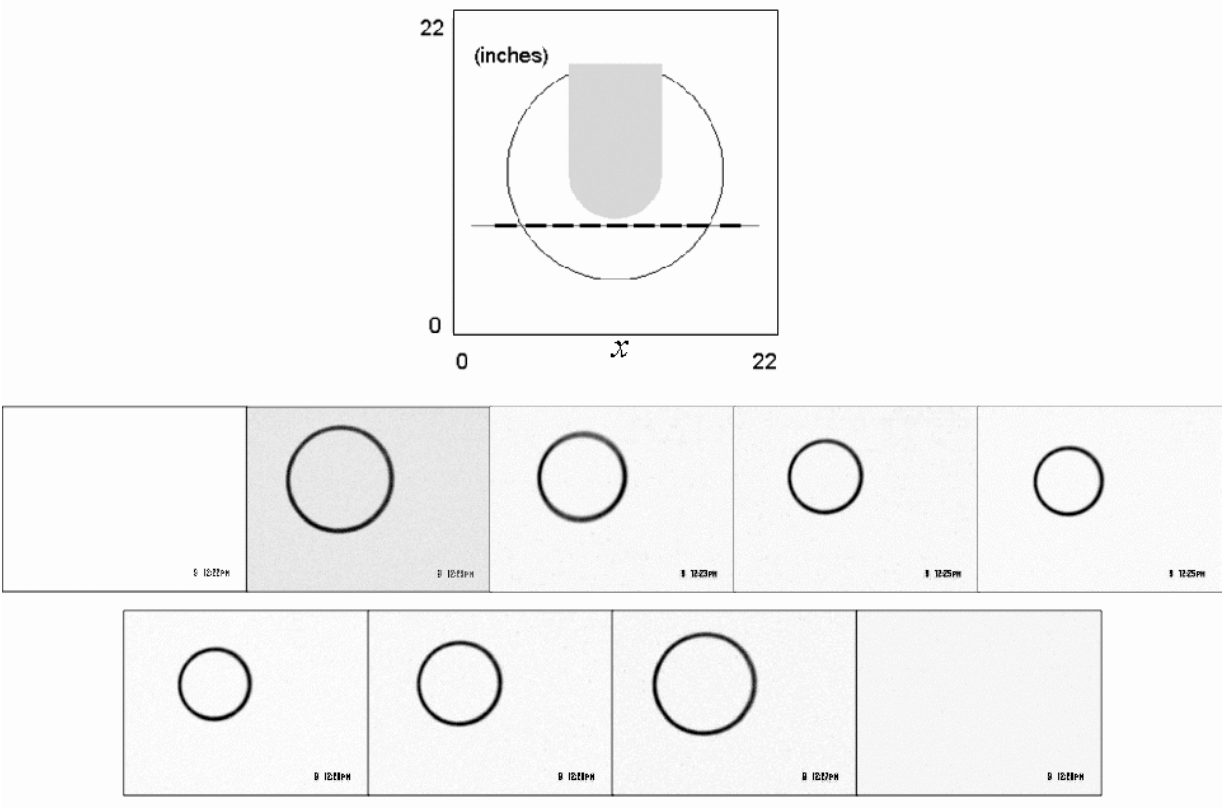

Figure 11.2: A tungsten lamp is placed at varying positions in front of the Howard University Raman Lidar telescope. The output from a fiber optic placed at the prime focus of the telescope illuminates a screen with the patterns shown. The first and last images show no indication of a pattern since these positions were outside of the telescope aperture. 


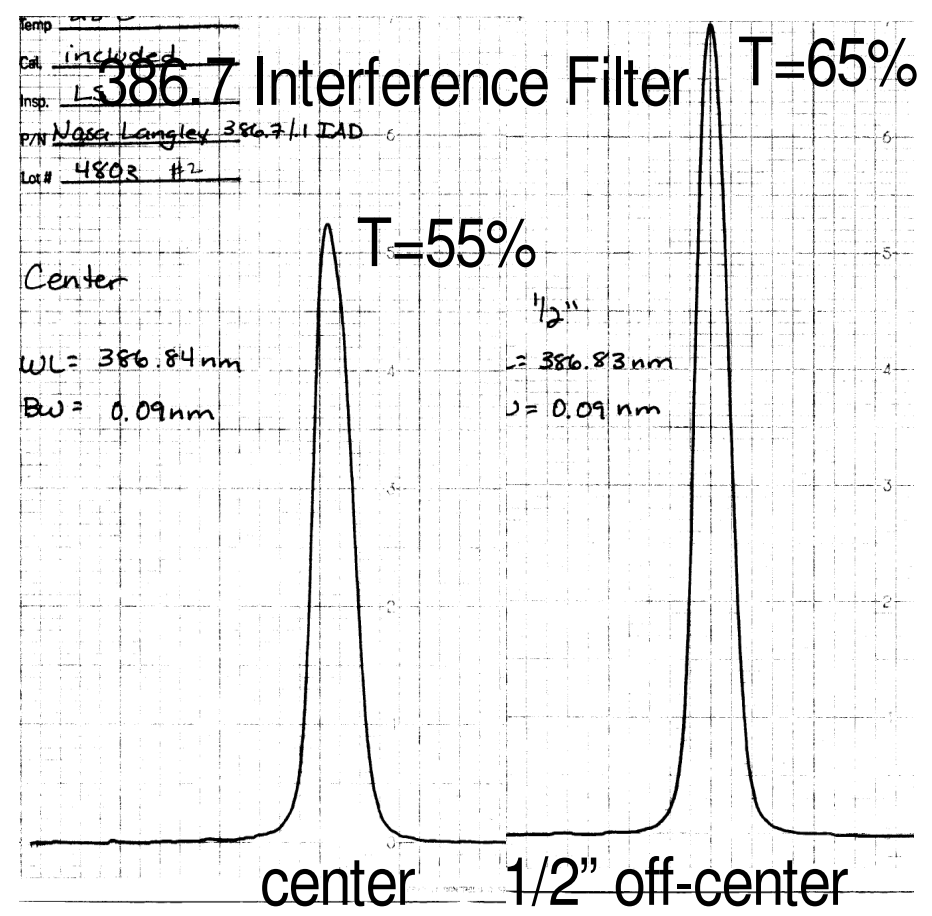

Figure 11.3: Bandpass of a $0.1 \mathrm{~nm}$ interference filter used to measure Raman scattering from atmospheric nitrogen. The transmission of the central $1 / 2^{\prime \prime}$ of the filter is approximately $55 \%$ while $1 / 2$ " off-center the transmission increases to $65 \%$. 

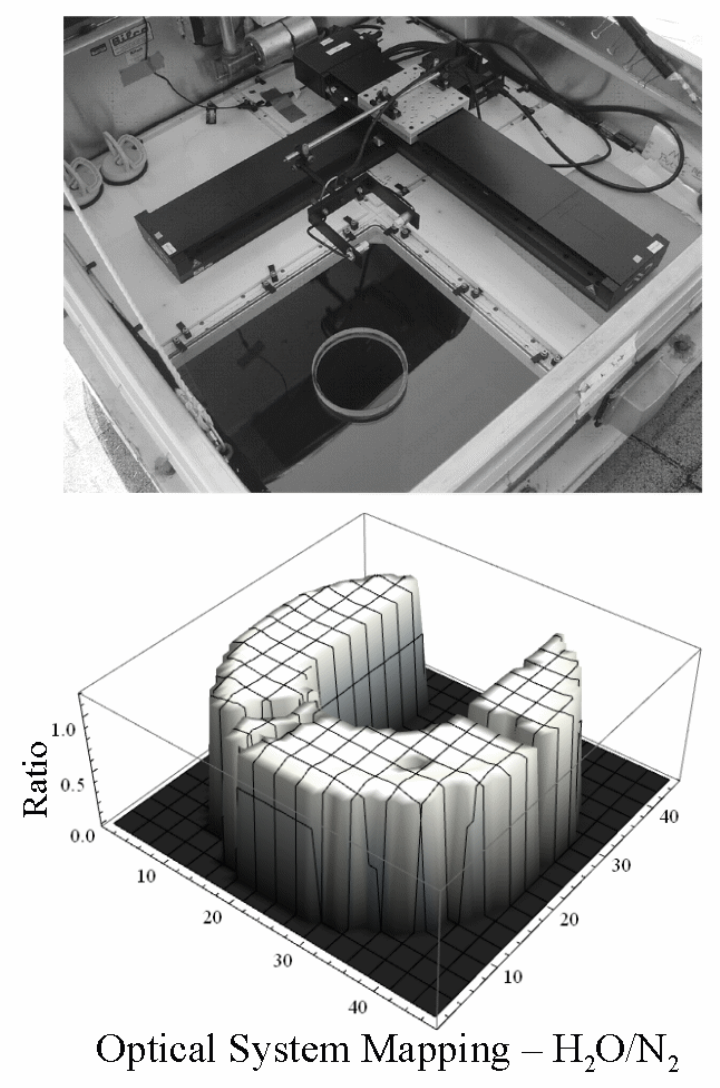

Figure 11.4: A system for translating a calibration lamp across the full aperture of a lidar receiver telescope is shown in the upper photo. A map of the ratio of total optical system efficiencies as a function of position across the telescope input aperture obtained with the translating calibration lamp is shown in the bottom of the figure. Dropouts due to a horizontal periscope, secondary spider veins and other obstructions are apparent in the mapping. 


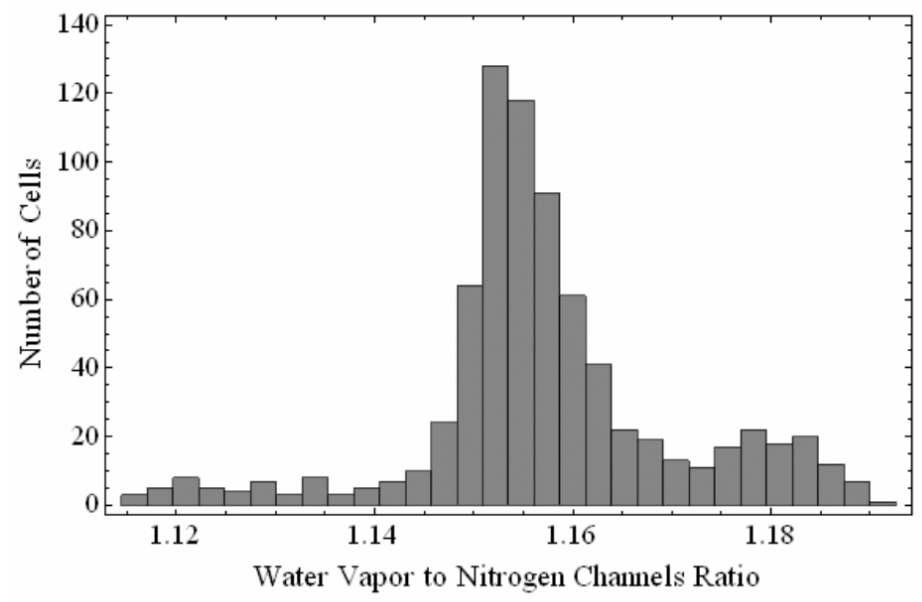

Figure 11.5: The histogram of the measured efficiency ratios recorded using a calibration lamp scanned over the full aperture of the Howard University Raman Lidar. The range of measured values is approximately $7 \%$. 

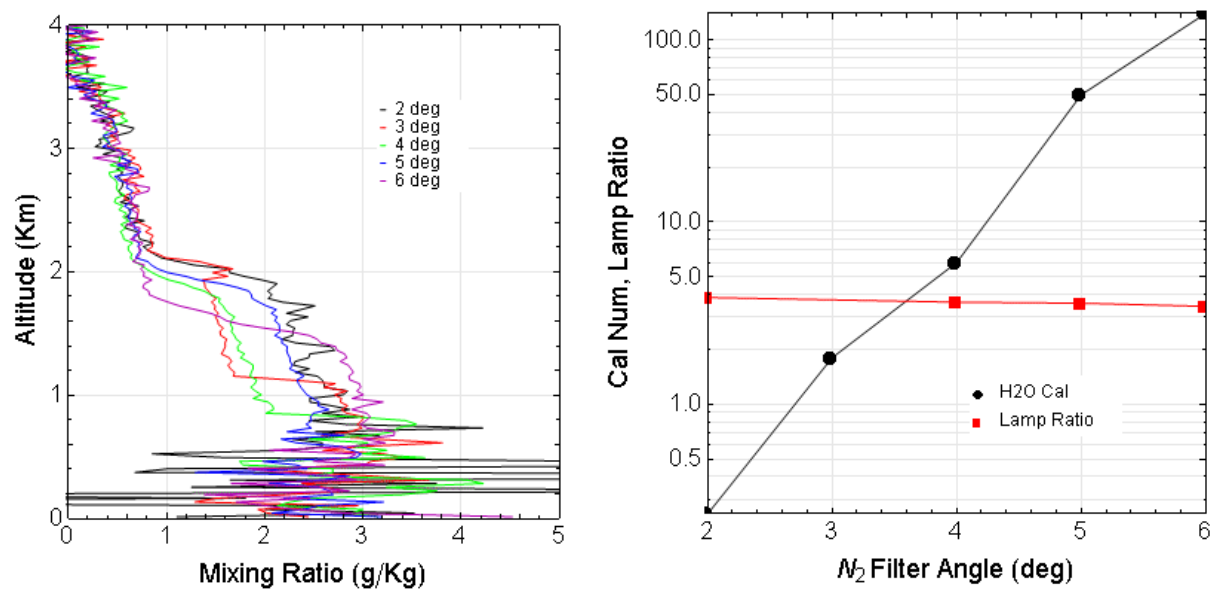

Figure 11.6: (Left) A series of water vapor mixing ratio profiles taken with the N2 filter at various tilt angles. The profiles have been individually calibrated. Efficiency ratios using a calibration lamp were quantified at each angle. (Right) The calibration number required for each of the profiles shown on the left along with the measured lamp ratios at each angle. No lamp data were acquired when the filter was tilted at an angle of 3 degrees. 